\title{
Properties of bulk liquid Pd and Pt and their free liquid surface studied with first principles techniques.
}

\author{
Beatriz G. del Rio, Luis E. González and David J. González \\ Departamento de Física Teórica, Universidad de Valladolid, Valladolid, SPAIN \\ E-mail: luisen@metodos.fam.cie.uva.es
}

\begin{abstract}
We have performed first principles computer simulations in order to study the structural and dynamic properties of bulk liquid Pd and Pt near their melting points. We find good agreement with the available experimental static structure and transport properties, and furthermore we provide more detailed information that is not available from experiments. Additional simulations have also been undertaken so as to study the free liquid surface of both liquid metals. The calculated longitudinal ionic density profile exhibits an oscillatory behavior whose properties have been analyzed. For both metals, the associated intrinsic surface structure factor presents a marked maximum related to surface layering.
\end{abstract}

PACS numbers: $61.20 . \mathrm{Ja}, 61.20 . \mathrm{Lc}, 61.25 . \mathrm{Mv}, 62.60+\mathrm{v}, 71.15 . \mathrm{Pd}$

Submitted to: Modelling Simul. Mater. Sci. Eng. 


\section{Introduction}

Platinum group metals, which include $\mathrm{Pt}$ and $\mathrm{Pd}$ as well as $\mathrm{Ru}, \mathrm{Rh}$, Os and Ir, are part of the so called precious metals. In the periodic table these systems are transition metals, with $4 d$ or $5 d$ orbitals incomplete. All of them share a low abundance at the Earth's crust, mostly due to their siderophile character that favors bonding with $\mathrm{Fe}$, and drives them to be located near the core rather than close to the surface of the Earth. All of them also share characteristics that make them important in industry and technology, including applications in jewelry, catalytic conversion in automobile exhausts, hydrogen storage and production in fuel cells, catalysis in crude oil refinement for production of gasoline, production of electronic devices and of glasses, coating for industrial crucibles, and medical applications such as their use in implants or in drug delivery for cancer treatment.

In this paper we concentrate on $\mathrm{Pd}$ and $\mathrm{Pt}$ in their liquid phase at ambient pressure. Their melting temperatures are rather high, 1828 and $2041 \mathrm{~K}$ respectively, and this is precisely one of the characteristics that make them interesting from a technological point of view. Also their density is very high, due to their small atomic radius (Os and Ir are the metals with highest mass density at room temperature).

Much in the same way that happens with other transition metals, the properties of the molten systems have not been thoroughly studied, either experimentally or from a theoretical perspective. For instance, we are only aware of one experimental determination of their structure factor, through x-ray diffraction (XD), performed by Waseda in the 1970's [1], who also reported the corresponding densities. The viscosity of liquid $\mathrm{Pt}(\mathrm{l}-\mathrm{Pt})$ and liquid $\mathrm{Pd}(\mathrm{l}-\mathrm{Pd})$ at melting have been reported in [2] and [3]. The sound velocity of l-Pt has also been reported in the same references, but not for l-Pd for which only estimates based on several correlations exist. As for the dynamical structure, no measurements have been performed yet up to our knowledge. On the theoretical side, the analysis of these two systems is also scarce, being limited to just two studies as far as we know. Both used classical molecular dynamics (CMD) with effective potentials to compute the static structure and some transport properties of the systems. Alemany et al [4] addressed both l-Pd and l-Pt using potentials obtained within the embedded atom method. Kart et al [5] considered l-Pd within the wider study of Pd-Ni alloys, using different effective potentials of the so called Sutton-Chen type.

The use of effective potentials allows the study of large simulation samples, but has an intrinsic limitation of accuracy related to the transfer of parameters from one thermodynamic state (for instance the solid at room temperature) to others of interest (for instance the liquid). This type of uncertainties are eliminated if the interatomic interactions are calculated from first principles, by taking into account explicitly the electronic degrees of freedom in the study. This is the case of ab-initio molecular dynamics (AIMD) methods. In particular, those based on density functional theory [6] (DFT) have become a very accurate technique for the study of the properties of condensed matter systems, in particular liquid metallic systems.

No such type of study has yet been undertaken for l-Pt or l-Pd, and this prompted us to perform AIMD simulations of these two systems in order to compute their static structure, their dynamic properties and also several transport properties of interest. We expect that these new accurate result may stimulate further work, experimental and/or theoretical, presently lacking.

Notice that $\mathrm{Pd}$ and $\mathrm{Pt}$ have the same number of valence electrons as $\mathrm{Ni}$, which 
Table 1. Thermodynamic states considered in this work for the bulk liquids. $\rho$ is the ionic number density, taken from [1] and $T$ is the temperature. $N_{c}$ is the total number of configurations.

\begin{tabular}{cccc}
\hline & $\rho\left(\AA^{-3}\right)$ & $T(\mathrm{~K})$ & $N_{c}$ \\
\hline $\mathrm{Pd}$ & 0.0594 & 1873 & 19500 \\
$\mathrm{Pt}$ & 0.0577 & 2053 & 19500 \\
\hline
\end{tabular}

has indeed be studied recently through the same AIMD method used here [7], and therefore it is also interesting to compare their properties with those of the latter.

In addition to the study of the bulk liquids we have also performed AIMD simulations in a slab configuration in order to address the properties of the free liquid surface (FLS) of both systems. Even if no experimental measurements of the surface structure of these melts have been undertaken yet, it is interesting to compare the results for l-Pd and l-Pt with those for which such experiments have indeed been performed, in order to find out similarities and differences with reported characteristics of liquid metallic surfaces.

The manuscript is organized as follows. In section 2 we briefly describe the AIMD simulation method and provide some technical details. The obtained results for the bulk liquids are reported in section 3, where they are compared with other previous studies as well as with the available experimental data. The results for the liquid-vapor interface are presented in section 4. Finally, some conclusions are drawn in section 5.

\section{Computational method}

The AIMD calculations for bulk l-Pd and l-Pt have been performed for thermodynamic states near their respective melting points (see Table 1). For each system, we have used 120 atoms in a cubic supercell with periodic boundary conditions (PBC). For both systems we have considered 10 valence electrons, including the $s, p$ and $d$ atomic orbitals. This amounts to 1200 valence electrons in the simulation cell.

The simulations were performed with the DFT based Quantum-ESPRESSO package [8]. The local density approximation (LDA), as parametrized by Perdew and Zunger [9], was used to account for the electronic exchange-correlation energy, and non-linear core corrections [10] were also included. The ion-electron interaction was described by ultrasoft pseudopotentials [11] which were generated from scalarrelativistic calculations of the atomic electron configurations $[\mathrm{Kr}] 4 \mathrm{~d}^{9} 5 \mathrm{~s}^{1}$ and $[\mathrm{Xe}] 5 \mathrm{~d}^{9} 6 \mathrm{~s}^{1}$ for $\mathrm{Pd}$ and $\mathrm{Pt}$ respectively.

The use of the LDA instead of other more sophisticated treatments of exchangecorrelation energy, is justified by its good performance for the solid fcc crystals of $\mathrm{Pd}$ and Pt. The comparison between experimental data for the lattice parameters of different solids with theoretical calculations using full potential linearized augmented plane waves and local orbitals and several flavours of exchange-correlation [12] proved that LDA was the best one in the case of $\mathrm{Pt}$ with an error about $0.01 \AA$, whereas a mismatch of only $0.03 \AA$ was produced with LDA in the case of Pd. Additional calculations for fcc Pd and Pt, using the same approach as followed in the present study (ultrasoft pseudopotentials and LDA) reduced the mismatch for both systems, yielding practically a perfect agreement with the experimental lattice constants and producing also quite reasonable results for other properties, such as the bulk modulus 
[13].

The initial atomic positions within the cell were chosen at random and the systems underwent a thermalization process lasting $15 \mathrm{ps}$ of simulation time. After this equilibration time the systems were clearly in the liquid phase, according to their disordered structure and their atomic diffusivity. Therefrom, microcanonical AIMD simulations were performed for 19500 time steps of 5.5 fs each, leading to a total simulation time of 107.25 ps for both systems. We have used a plane-wave representation with an energy cutoff of 25.0 Ryd and the single $\Gamma$ point was used for sampling the Brillouin zone. These final 19500 equilibrium configurations were subsequently used for the evaluation of the static, dynamic and electronic properties of bulk l-Pd and l-Pt. This large number of configurations is essential in order to obtain well converged liquid properties, especially dynamic ones. In addition to the large computational demands of first principles calculations, this required long simulation time poses constraints on the feasible size of the simulation samples, especially on the total number of valence electrons. The samples considered here, with 1200 electrons, are quite typical in state-of-the-art liquid ab initio studies. Finally, we emphasize that the present AIMD simulation method has already provided an accurate description of several static and dynamic properties of bulk l-Sn, l-Hg, l-Cd, l- Ag, l-Ni and l-Ti $[7,14,15,16,17,18]$.

Another set of AIMD calculations was carried out in order to study the FLS of l-Pd and l-Pt at temperatures of 2000 and $2200 \mathrm{~K}$ respectively, which are slightly higher than the previous bulk calculations, while the initial densities were taken the same as in Table 1. For this study we have used a slab geometry with 120 atoms (and 1200 valence electrons) in a supercell with two free surfaces normal to the $z$ axis. The dimensions of the slab were initially $L_{x} \times L_{y} \times d_{z}$ where $L_{x}=L_{y}=10.71 \AA$ and $d_{z}$ is such that the initial density is recovered (17.645 $\AA$ for l-Pd and $18.165 \AA$ for l-Pt). The simulation box, replicated periodically, had sizes $L_{x} \times L_{y} \times L_{z}$, with $L_{z}=26.60$ $\AA$. This corresponds to a vacuum around $8 \AA$ wide separating the periodic images of the slab along the $z$ axis, which is enough to allow neglecting inter-slab interactions.

During its evolution, the slab may contract or expand so as to achieve the zero external pressure condition; more details can be found in Refs. [19, 20, 21, 22, 23]. The initial atomic positions within the slab were taken at random and both systems were thermalized during $20 \mathrm{ps}$ of simulation time. Therefrom, microcanonical AIMD simulations were performed over $28500(\mathrm{Pd})$ and $32500(\mathrm{Pt})$ time steps. The same considerations mentioned for the bulk systems about simulation time and system size are obviously valid in the slab configuration too. Also note that this computational scheme has already been used to study the FLS of l-Sn, l-Hg, l-Cd and l-Ag, yielding a good description of the available experimental x-ray reflectivity data [16, 23].

\section{Results: Bulk Properties}

Several bulk liquid properties have been calculated, both static and dynamic. Those correlation functions that have a dependence on the wavevector, $\vec{q}$, have been converted into module dependent quantities, as corresponds to isotropic systems. This has been done by averaging over the different vectors allowed by the $\mathrm{PBC}$ with the same module $q \equiv|\vec{q}|$. 


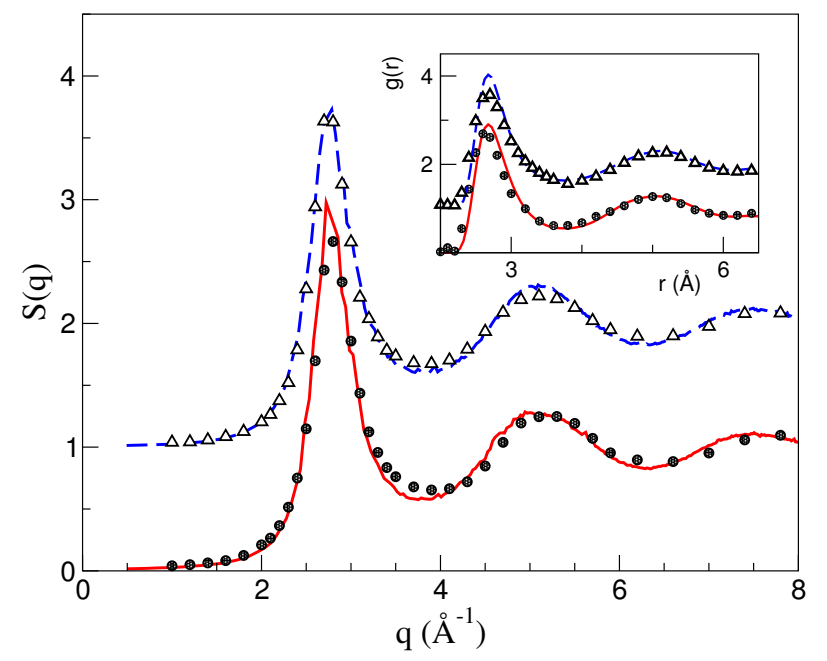

Figure 1. Static structure factor, $S(q)$, of l-Pd and l-Pt at 1873 and $2053 \mathrm{~K}$ respectively. Full line: AIMD calculations for Pd. Broken line: AIMD results for Pt. Circles: experimental XD data for Pd from Waseda [1]. Triangles: experimental XD data for $\mathrm{Pt}$ from Waseda [1]. The inset shows the corresponding results for the pair distribution function $g(r)$. The results for Pt have been shifted upwards by 1 unit for clarity.

\subsection{Static properties}

Figure 1 shows the comparison between our AIMD results for the static structure factor, $S(q)$, and the pair distribution function, $g(r)$, with the XD data of Waseda [1]. The calculated $S(q)$ 's for l-Pd and l-Pt show an excellent agreement with the experimental data. They have symmetric main peaks located respectively at $q_{p}=2.79$ and $2.77 \AA^{-1}$, and their shapes and amplitudes practically coincide with experiment. There is ony a very small phaseshift between the experimental and the AIMD subsequent oscillations in the case of l-Pt. A good agreement is also found for the calculated $g(r)$. The coordination number, evaluated through the integration of $4 \pi r^{2} g(r)$ up to the position of its first minimum $\left(R_{\min }=3.77 \AA\right.$ for both l-Pd and l-Pt), takes the value of $\approx 12.8$ atoms for both systems. This is a typical value for simple liquid metals near their triple point [24].

A more detailed, three dimensional, description of the local order in the liquid can be achieved by resorting to the common neighbor analysis (CNA) method [25], which provides information about the local environment around a pair of neighbouring atoms. Each type of configuration around a pair is characterized by four indices and the relative abundances of the different types pinpoint to specific local structures, such as bcc environments (144x and 166x pairs), perfect or distorted icosahedra (icos) (155x, 154x and 143x pairs), close-packed structures (142x pairs), and other, more complex, polytetrahedral environments.

Starting from a few configurations generated in the simulation, we first removed the thermal noise by finding the corresponding inherent structures, i.e. the local minima in the potential energy landscape closest to the corresponding configurations. Then we performed the CNA calculation in these states, and the averaged results 
Liquid palladium and platinum: bulk and surface properties.

Table 2. Common neighbour analysis of the AIMD configurations of 1-Pd and l-Pt compared with the values corresponding to several perfect local structures.

\begin{tabular}{llllllllll}
\hline Pairs & 1551 & 1541 & 1431 & 1321 & 1421 & 1422 & 1311 & 1441 & 1661 \\
\hline l-Pd & 0.24 & 0.22 & 0.21 & 0.03 & 0.04 & 0.06 & 0.03 & 0.05 & 0.06 \\
\hline l-Pt & 0.14 & 0.21 & 0.22 & 0.05 & 0.07 & 0.10 & 0.06 & 0.05 & 0.04 \\
\hline icos & 1.00 & 0.00 & 0.00 & 0.00 & 0.00 & 0.00 & 0.00 & 0.00 & 0.00 \\
\hline hcp & 0.00 & 0.00 & 0.00 & 0.00 & 0.50 & 0.50 & 0.00 & 0.00 & 0.00 \\
\hline fcc & 0.00 & 0.00 & 0.00 & 0.00 & 1.00 & 0.00 & 0.00 & 0.00 & 0.00 \\
\hline bcc & 0.00 & 0.00 & 0.00 & 0.00 & 0.00 & 0.00 & 0.00 & 0.43 & 0.57 \\
\hline
\end{tabular}

are summarized in Table 2. First, we observe that the five-fold symmetry has an overwhelming presence in both l-Pd $(\approx 67 \%)$ and l-Pt $(\approx 57 \%)$. Also the fcc/hcp structures have a sizeable contribution $(\approx 17 \%$ in l-Pt and $\approx 10 \%$ in l-Pd), whereas bcc-like local structures amount to around $10 \%$ in both systems. Comparing these results with those of liquid $\mathrm{Ni}[7]$, we find that there is an increasing fraction, from 6 to 10 to $17 \%$, of close-packed structures when moving down the series Ni-Pd-Pt. Note that the three systems melt from an fcc phase. On the contrary, the amount of five-fold symmetry structures, even if dominant, decreases from 70 to 67 to $57 \%$. Finally, the fraction of atoms in a bcc-like environment keeps roughly constant in the series.

The long wavelength limit, $q \rightarrow 0$, of the structure factor is directly related to the isothermal compressibility, $\kappa_{T}$, through the well known relation $S(0)=\rho k_{B} T \kappa_{T}$, where $k_{B}$ is Boltzmann's constant. We have extrapolated the AIMD $S(q)$ to the long wavelength limit using a quadratic function, leading to compressibilities $\kappa_{T}^{\mathrm{AIMD}}(\mathrm{Pd})$ $=9.1 \pm 0.4 \mathrm{TPa}^{-1}$ and $\kappa_{T}^{\mathrm{AIMD}}(\mathrm{Pt})=6.7 \pm 0.5 \mathrm{TPa}^{-1}$. Several estimates for this magnitude at the melting point have been proposed by Marcus [26] and Blairs [2] using different semiempirical formulas that relate the isothermal compressibility to other thermophysical magnitudes. Additionally, an experimental value for l-Pt has also been reported by Blairs, based on measurements of the sound velocity, the mass density and the ratio between specific heats, $\gamma=C_{P} / C_{V}$, and this can also serve as an indicator of the accuracy of the estimates for both metals. The results for 1Pt are $\kappa_{T}^{\text {Marcus }}(\mathrm{Pt})=10.2 \mathrm{TPa}^{-1}, \kappa_{T}^{\text {Blairs }}(\mathrm{Pt})=10.0 \mathrm{TPa}^{-1}$, and $\kappa_{T}^{\text {exp }}(\mathrm{Pt})=8.4 \mathrm{TPa}^{-1}$, indicating some overestimation in the semiempirical estimates, which basically agree between themselves. Note that our value is closer to the experimental result than to the estimates. In the case of l-Pd there are no experimental data, while the estimates are $\kappa_{T}^{\text {Marcus }}(\mathrm{Pd})=12.8 \mathrm{TPa}^{-1}, \kappa_{T}^{\text {Blairs }}(\mathrm{Pd})=11.7 \mathrm{TPa}^{-1}$, which show a somewhat larger disagreement between each other. If we assume that these values also suffer of some overestimation, then our AIMD result seems also reasonable.

\subsection{Dynamic properties}

3.2.1. Collective dynamics The collective dynamics of density fluctuations in a liquid is described by the intermediate scattering function, $F(q, t)$, which is the autocorrelation function of the microscopic wavevector dependent density [24]. The Fourier Transform (FT) of $F(q, t)$ into the frequency domain leads to the dynamic 


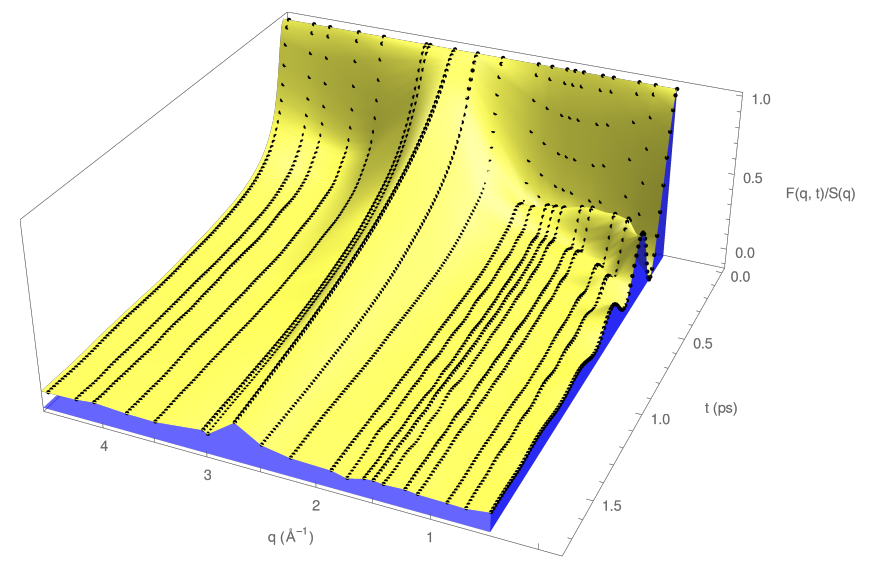

Figure 2. Normalized intermediate scattering functions, $F(q, t) / S(q)$, at several $q$-values, for l-Pd at $1853 \mathrm{~K}$.

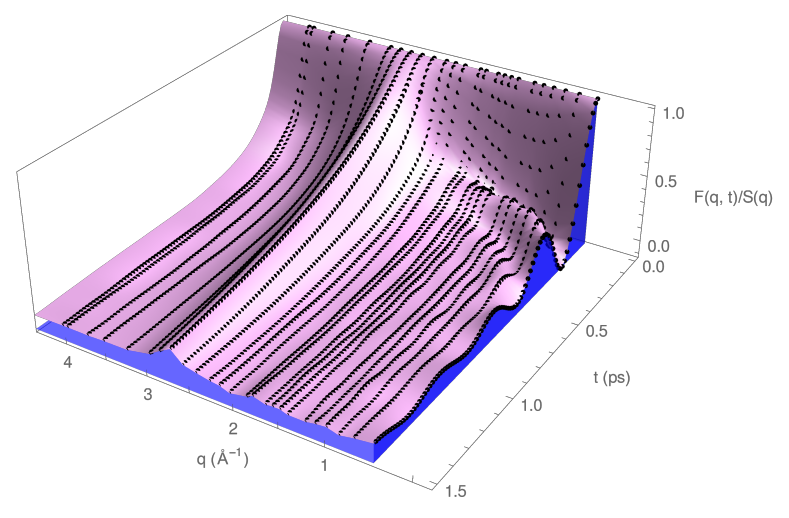

Figure 3. Same as the previous figure, but for l-Pt at $2053 \mathrm{~K}$.

structure factor, $S(q, \omega)$. Another basic dynamic collective magnitude is the current due to the overall motion of the particles [24]. Being a vector quantity, it can be split into its longitudinal and transverse components with respect to $\vec{q}$. Therefrom, the longitudinal, $J_{L}(q, t)$, and transverse $J_{T}(q, t)$, current correlation functions are obtained as autocorrelation functions of the corresponding quantities [24]. Their time FT yields the associated spectra, $J_{L}(q, \omega)$ and $J_{T}(q, \omega)$ respectively.

Figures 2-3 depict, for a range of $q$-values, the AIMD results for $F(q, t) / S(q)$ in l-Pd and l-Pt respectively. For small $q$-values, the $F(q, t)$ show clear oscillations, indicative of wave propagation, superimposed onto a globally decaying form, indicative of relaxation modes. The relative weight of these two terms changes upon increasing $q$, and at some point around $0.6 q_{p}$ the relaxation term overweighs the propagating one, leading at $q \approx q_{p}$ to the very slow monotonic decay of $F(q, t)$ known as "de Gennes narrowing".

In order to understand the physical origins of the propagation and relaxation mechanisms indicated above it is necessary to resort to theoretical models for the 
dynamics of liquids. Such theories [24, 27] are most effectively stated in terms of the memory functions of $F(q, t)$, in particular its second order memory function, $N(q, t)$. This function is most easily defined in the Laplace domain: the Laplace Transforms of $F(q, t)$ and $N(q, t)$ are related by the equation

$$
\tilde{F}(q, z)=S(q)\left(z+\frac{M_{0}(q)}{z+\tilde{N}(q, z)}\right)^{-1},
$$

where $M_{0}(q)=k_{B} T q^{2} /(m S(q))$, with $m$ denoting the atomic mass.

In the long wavelength limit, and for small $q$, liquids behave according to the hydrodynamics model of macroscopic fluid dynamics, which can be formulated as the decomposition of $N(q, t)$ into a sum of an instantaneous (Dirac-delta) decay function associated to longitudinal viscous relaxation and a slower decay function (exponential) associated to thermal relaxation.

For increasing $q$ values the hydrodynamic model loses validity and can be replaced by a different one where $N(q, t)$ is now described as a sum of two exponentially decaying functions, a fast one and a slower one. In a generalized hydrodynamic picture the fast decay would be associated to the viscoelasticity of the liquid, and the slower decay would be ascribed to thermal relaxation. However the opposite identification has also been suggested for some liquid metals [28], based on an analysis of experimental inelastic x-ray scattering data for liquid Li. In this generalized viscoelastic model thermal relaxation should be ascribed to the fast decay term and viscolastic relaxation to the slow one. The amplitudes and relaxation times associated to each of the two physically distinct terms are given in terms of $q$-dependent generalizations of thermophysical quantities [27]. In the thermal term we find the $q$-extensions of $\gamma$, previously defined, and of the thermal diffusivity $D_{T}$. In the viscoelastic part we have the $q$-dependent generalizations of elastic and longitudinal viscous coefficients, and again that of $\gamma$.

The $N(q, t)$ have been calculated from the AIMD $F(q, t)$, then fitted to a sum of two exponentially decaying terms (fast and slow),

$$
N(q, t)=A_{f}(q) \exp \left[-t / \tau_{f}(q)\right]+A_{s}(q) \exp \left[-t / \tau_{s}(q)\right],
$$

and finally analyzed in order to find out whether they are consistent with a generalized hydrodynamic model (fast viscoelastic mode and a slow thermal one), or a generalized viscoelastic model, where the fast term is the thermal one. In order to do so, have chosen to evaluate the generalized heat capacity ratio, $\gamma(q)$. The values for $\gamma(q)$ are obtained from the amplitude of the slow mode, $A_{s}(q)$, but the formula that relates them is different depending on the physical channel (thermal or viscoelastic) ascribed to the slow mode. Thus, we have calculated the functions $\gamma_{\text {th }}(q)$ and $\gamma_{v}(q)$ which represent the values obtained for $\gamma(q)$ when either the thermal or the viscoelastic relaxations proceed through the slow mode. The results are plotted in Figure 4.

For l-Pd, it is observed that $\gamma_{v}(q)$ shows a monotonous increasing behaviour from the value of 1.94 that it takes at the smallest attainable $q$-value in the simulation, $q_{\min } \approx 0.497 \AA^{-1}$. On the other hand, $\gamma_{\text {th }}(q)$ starts from a value $\gamma_{\text {th }}\left(q_{\text {min }}\right) \approx 1.29$ and slowly decreases towards values $\approx 1.0$ in the $q$-range shown in the figure. A very similar behavior is obtained for l-Pt: at $q_{\min } \approx 0.492 \AA^{-1}$, we obtained $\gamma_{v}\left(q_{\min }\right) \approx 2.05$, followed by an increasing behaviour with $q$, while on the other hand, we have $\gamma_{\text {th }}\left(q_{\text {min }}\right) \approx 1.40$ and it smoothly decreases towards values $\approx 1.0$.

We are not aware of any experimental data for the $\gamma(q)$ in l-Pd and l-Pt, but we have compared with the results obtained for other liquid metals[15, 16, 29], which 


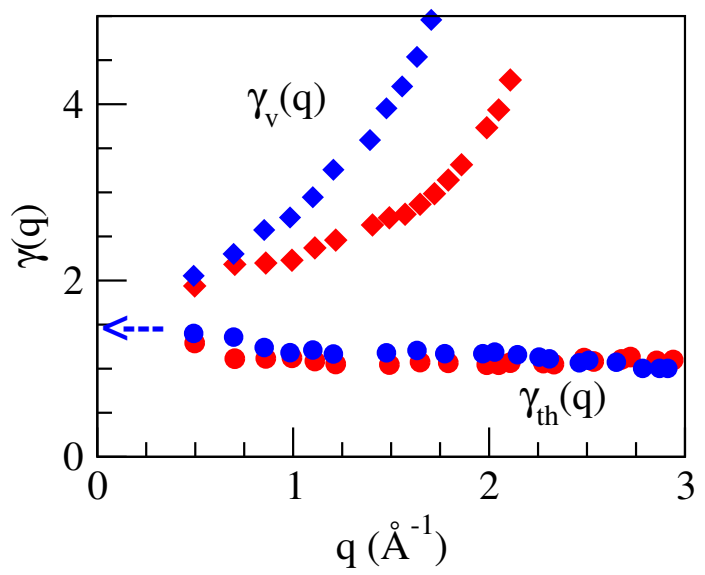

Figure 4. Generalized specific heat ratio, $\gamma(q)$, as obtained from the generalized hydrodynamic model (circles) and the generalized viscoelastic model (lozenges). The red (blue) symbols correspond to $\mathrm{Pd}(\mathrm{Pt})$. The blue arrow shows the experimental value of $\gamma=1.51$ for l-Pt reported by Blairs [2].

yielded $\gamma(q)$ curves that show a continuous decrease from the initial value $\gamma_{0}$ towards values around 1.0 at $q_{p} / 2$ and then there is a smooth increase with a maximum at $\approx q_{p}$. Similar behavior has been found in the "experimental" data of Hosokawa et al [30] for the $\gamma(q)$ of l-Fe near melting. Finally, we note that Blairs [2] reported for l-Pt an experimental macroscopic value $\gamma=1.51$, which is in reasonable agreement with the extrapolation to the long wavelength limit of our calculated value $\gamma_{\operatorname{th}}(q)$. Therefore, based on these AIMD results and on previously observed trends in various liquid metals, we can conclude that the slow mode is that of thermal relaxation, making the generalized hydrodynamic model as the appropriate one for describing the microscopic dynamics of both l-Pd and l-Pt near their respective triple point. Note that this is in line also with the previous analysis of the data for l-Ni [7].

Figures 5-6 show the behavior of the dynamic structure factors, $S(q, \omega)$, calculated as the FT of the $F(q, t)$. For both systems, the obtained $S(q, \omega)$ show a similar behaviour, namely, existence of side-peaks, indicative of collective density excitations, up to $q \approx(3 / 5) q_{p}$; therefrom the side-peaks evolve into shoulders which last up to $q \approx(4 / 5) q_{p}$ and for greater $q$-values the corresponding $S(q, \omega)$ show a monotonic decreasing behavior.

We have also evaluated the current correlation functions and the associated spectra. The AIMD calculated $J_{L}(q, \omega)$ exhibit a peak for each $q$-value and the frequencies of those peaks give rise to the dispersion relation of the longitudinal modes, $\omega_{L}(q)$, which is plotted in Figure 7. The $\omega_{L}(q)$ exhibits a behaviour typical of liquid systems with a minimum located at $\approx q_{p}$. In the low- $q$ region, the slope of the $\omega_{L}(q)$ curve gives the bulk adiabatic sound velocity, $c_{s}$. Using the calculated frequencies, $\omega_{L}(q)$, corresponding to the region $q \leq 1.2 \AA^{-1}$, we have estimated its slope and obtained $c_{s} \approx 3450 \pm 150 \mathrm{~m} / \mathrm{s}(\mathrm{l}-\mathrm{Pd})$ and $c_{s} \approx 3000 \pm 150 \mathrm{~m} / \mathrm{s}(\mathrm{l}-\mathrm{Pt})$. We are not aware of any experimental data for $c_{s}$ of l-Pd, but Iida and Guthrie [3] have suggested a value $c_{s} \approx 3586 \mathrm{~m} / \mathrm{s}$ which was obtained by using an empirical expression relating several experimental thermophysical quantities. As for l-Pt, the present AIMD result compares well with the experimental data at melting, namely $c_{s}=3053 \mathrm{~m} / \mathrm{s}[2]$. 


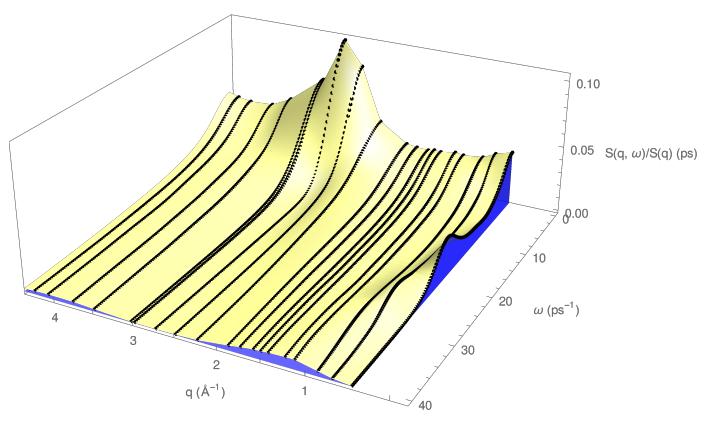

Figure 5. Dynamic structure factors, $S(q, \omega)$, at several $q$-values, for l-Pd at $1853 \mathrm{~K}$

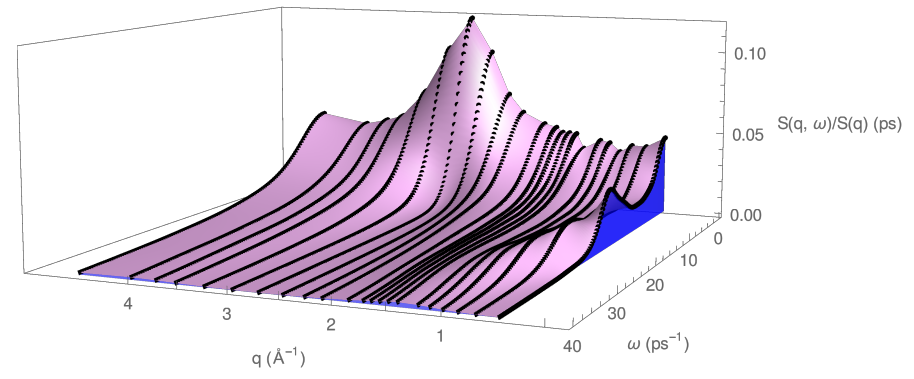

Figure 6. Same as the previous figure, but for l-Pt at $2053 \mathrm{~K}$.

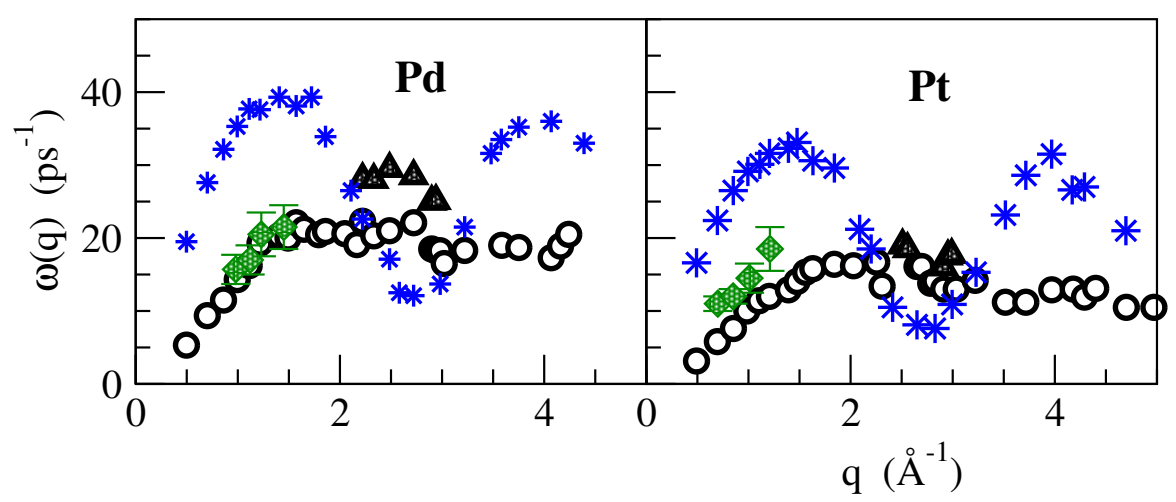

Figure 7. Dispersion relations for l-Pd at $1873 \mathrm{~K}$ and for l-Pt at $2053 \mathrm{~K}$. Stars: Longitudinal dispersion obtained from the positions of the peaks in the spectra $J_{L}(q, \omega)$. Circles and triangles: Transverse dispersion from the positions of the peaks in the spectra $J_{T}(q, \omega)$. The lozenges with error bars are the positions of the transverse-like excitation modes (see text) found in the calculated dynamic structure factors, $S(q, \omega)$.

The transverse current correlation function, $J_{T}(q, t)$, provides information on the shear modes. Its spectrum, $J_{T}(q, \omega)$, when plotted as a function of $\omega$, may display 


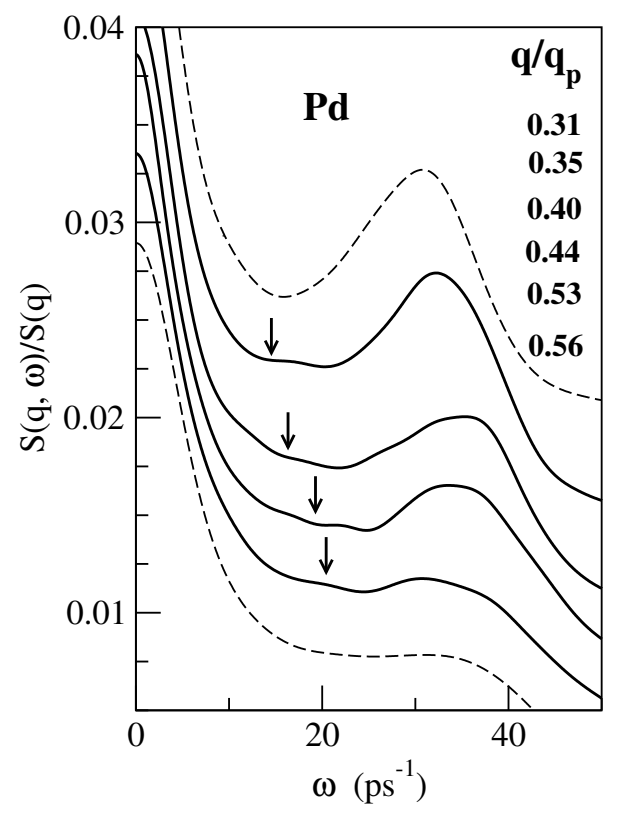

Figure 8. AIMD calculated dynamic structure factors $S(q, \omega) / S(q)$ of l-Pd at $1873 \mathrm{~K}$ and several $q$-values. The vertical scales are offset for clarity. The arrows point to the locations of the maxima in the $J_{T}(q, \omega)$ at the corresponding $q$-value.

peaks, within some $q$-range, which are related to propagating shear waves. For both l-Pd and l-Pt, we have found that the corresponding $J_{T}(q, \omega)$ already shows a peak for the smallest attainable $q$-value, i.e. $q_{\min }=0.18 q_{p}$ and it lasts up to $q \leq 3 q_{p}$, which is a usual trend in most simple liquid metals [24].

From the position of the peaks in the $J_{T}(q, \omega)$, the associated dispersion relation for the transverse modes has been obtained as shown in Figure 7. For both metals, we have found two branches with the high-frequency branch existing over a limited $q$-range around the position of the main peak of the $S(q)$. To our knowledge, the emergence of a second, high-frequency, transverse branch has been found in high pressure l-Li, l-Na, l-Fe, l-Pb and l-Al [31, 32, 33, 34], but recently some AIMD studies at ambient pressure have also unveiled a second high-frequency transverse branch, in particular for $\mathrm{l}-\mathrm{Ni}$, l-Tl, l-Zn and l-Sn near melting [7, 35, 36, 37]. In all cases, this new branch appears for $q$-values greater than the first pseudo-Brillouin zone boundary, i.e. $q>q_{p} / 2$, and its physical origin has been suggested to be the indirect coupling of longitudinal and transverse currents, as described by so-called mode-coupling theories [36, 37].

From our obtained AIMD results for $J_{T}(q, t)$, the shear viscosity coefficient, $\eta$, has been calculated. The method proceeds through the calculation of a $q$-dependent (generalized) shear viscosity, $\eta(q)$, from the time integral of $J_{T}(q, t)$, followed by extrapolation to the long wavelength limit, which leads to $\eta$. Additional details are given in References $[24,38,39]$. For l-Pd, we have obtained $\eta_{\mathrm{AIMD}}=4.57 \pm 0.15$ $\mathrm{GPa} \cdot \mathrm{ps}$ which compares well with the corresponding experimental value (at melting) $\eta_{\exp }=4.22 \pm 0.15 \mathrm{GPa} \cdot \mathrm{ps}[3]$; moreover, we note that previous CMD simulation studies have yielded smaller values, i.e. $3.68 \pm 0.50 \mathrm{GPa} \cdot \mathrm{ps}[4]$ and $2.91 \pm 0.86 \mathrm{GPa} \cdot \mathrm{ps}$ [5]. As for l-Pt, there are two published experimental data, $\eta_{\exp }=4.82$ and 6.74 


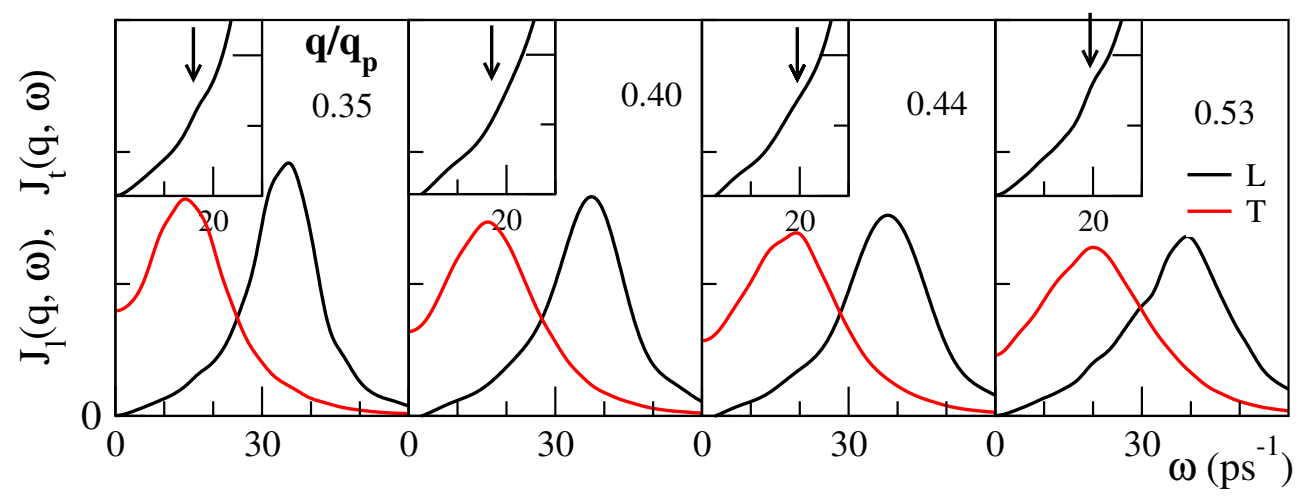

Figure 9. AIMD calculated longitudinal and transverse currents spectral functions, $J_{L}(q, \omega)$ and $J_{T}(q, \omega)$ respectively, for l-Pd at several $q / q_{p}$ values. The insets in the graphs provide a closer view of the small $\omega$ region of the $J_{L}(q, \omega)$ and the arrows point to the locations of the transverse-like excitations in the $S(q, \omega)$.

GPa.ps [3], with the smaller one being very close to the present calculation, which is $\eta_{\mathrm{AIMD}}=4.90 \pm 0.25 \mathrm{GPa} \cdot \mathrm{ps}$. A previous CMD simulation of l-Pt [4] yielded $6.08 \pm 0.95$ GPa.ps [4], closer to the larger experimental data.

Recently, signatures of possibly transverse-like low energy excitations have been observed, either in experiments or in AIMD simulations, as weak shoulders located in the region between the quasielastic and the longitudinal inelastic peaks of the $S(q, \omega)$. These excitations are usually visible within a small $q$-range around $q_{p} / 2$, since for smaller $q$-values they can be overcome by the wide quasielastic peak centered at $\omega=0$, whereas for larger $q$-values they can be covered by the also increasingly wide inelastic peaks. These excitations were first detected in the inelastic x-ray scattering spectra of l-Ga [40] at $313 \mathrm{~K}$. Subsequently they have also been observed in l-Cu, l-Sn, l-Zn, l-Na, l-Fe and l-Ni [7, 36, 37, 41, 42, 43].

To check their presence in l-Pd and l-Pt we have analyzed our $S(q, \omega)$ results and found some weak features in the $\omega$-region between the quasielastic and inelastic peaks. These are more noticeable for l-Pd and are plotted in Figure 8 for the $q$-range 1.00 $\AA^{-1} \leq q \leq 1.50 \AA^{-1}$ where they are visible. Furthermore, as $J_{L}(q, \omega)=(\omega / q)^{2} S(q, \omega)$, these excitations are also expected to be observable in the $J_{L}(q, \omega)$. Therefore, we have plotted in figure 9 the AIMD results for $J_{L}(q, \omega)$ and $J_{T}(q, \omega)$. Notice that the $J_{L}(q, \omega)$ display weak shoulders at frequencies close to those associated with the weak features present in the respective $S(q, \omega)$, and that these frequencies are also close to the frequencies where the $J_{T}(q, \omega)$ shows a peak. This is in fact the reason to assign a transverse-like character to them. The appearance of these, possibly transverselike, excitations in the $S(q, \omega)$ of single component liquid metals remains an unsolved problem and, as far as we know, neither models nor explanations of its origin have been put forward yet.

3.2.2. Single particle dynamics Two basic functions related to single-particle dynamics in a system are the mean square displacement, $\delta R^{2}(t)=\left\langle\left|\vec{R}_{1}(t)-\vec{R}_{1}(0)\right|^{2}\right\rangle$, and the (normalized) velocity autocorrelation function, $Z(t)=\left\langle\vec{v}_{1}(t) \cdot \vec{v}_{1}(0)\right\rangle /\left\langle v_{1}^{2}\right\rangle$. In the previous definitions the index 1 denotes a tagged ion in the fluid. The self-diffusion 


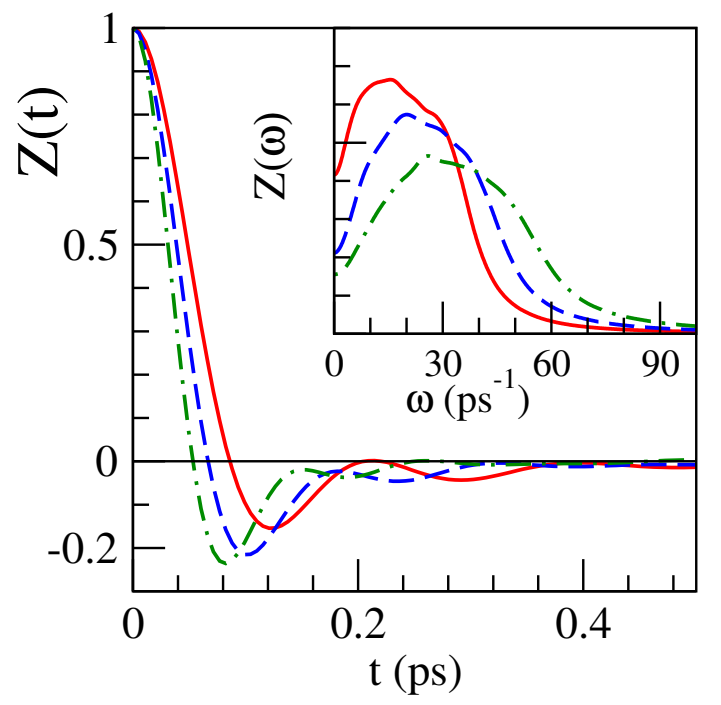

Figure 10. Normalized AIMD calculated velocity autocorrelation function of l-Pt (red full line), l-Pd (blue dashed line) and l-Ni (green dash-dotted line) near their respective melting points. The inset represents the corresponding power spectrum $Z(\omega)$.

coefficient, $D$, can be obtained from both functions, either in terms of the slope of $\delta R^{2}(t)$ for long times, or in terms of the time integral of $Z(t)$. The values obtained from our AIMD simulations through both routes are identical within the statistical accuracy, and are by chance numerically equal for both systems, $D_{\mathrm{AIMD}}(\mathrm{Pd})=D_{\mathrm{AIMD}}(\mathrm{Pt})=$ $0.27 \pm 0.02 \AA^{2} / \mathrm{ps}$. There are no experimental data to compare with. Previous CMD studies produced values of $0.40 \AA^{2} / \mathrm{ps}$ [4] and $0.50 \AA^{2} / \mathrm{ps}$ [5] for l-Pd, and $0.28 \AA^{2} / \mathrm{ps}$ [4] for l-Pt.

The velocity autocorrelation function also provides information about the distribution of atomic vibration frequencies through its FT, $Z(\omega)$, called the power spectrum. We show in figure 10 the calculated $Z(t)$ and the corresponding power spectrum for l-Pd, l-Pt, and additionally we have also included those corresponding to l-Ni [7]. The $Z(t)$ exhibit the typical backscattering behavior with a first minimum followed by rather weak oscillations and the $Z(t)$ always remaining negative. Notice that the $Z(t)$ are qualitatively very similar and the differences are just those related to the increasing ionic mass when going from l-Ni to l-Pt. As for the $Z(\omega)$, we observe a single peak followed by a weak shoulder which is more marked for l-Pt. We mention that the ab-initio study of 1-Tl [35] also found a similar structure for the corresponding $Z(\omega)$, although the shoulder was more marked. Moreover, they suggested a correlation between the appearance of a high frequency shoulder/peak in the $Z(\omega)$ and the emergence of a second high-frequency transverse branch with practically the same frequency as that of the peak/shoulder. Indeed, that correlation is also shown in the present AIMD calculations for l-Pd and l-Pt.

\subsection{Electronic properties}

We have also calculated the partial and total electronic density of states per atom, $n(E)$. It was obtained from the self-consistent eigenvalues and it was averaged over 


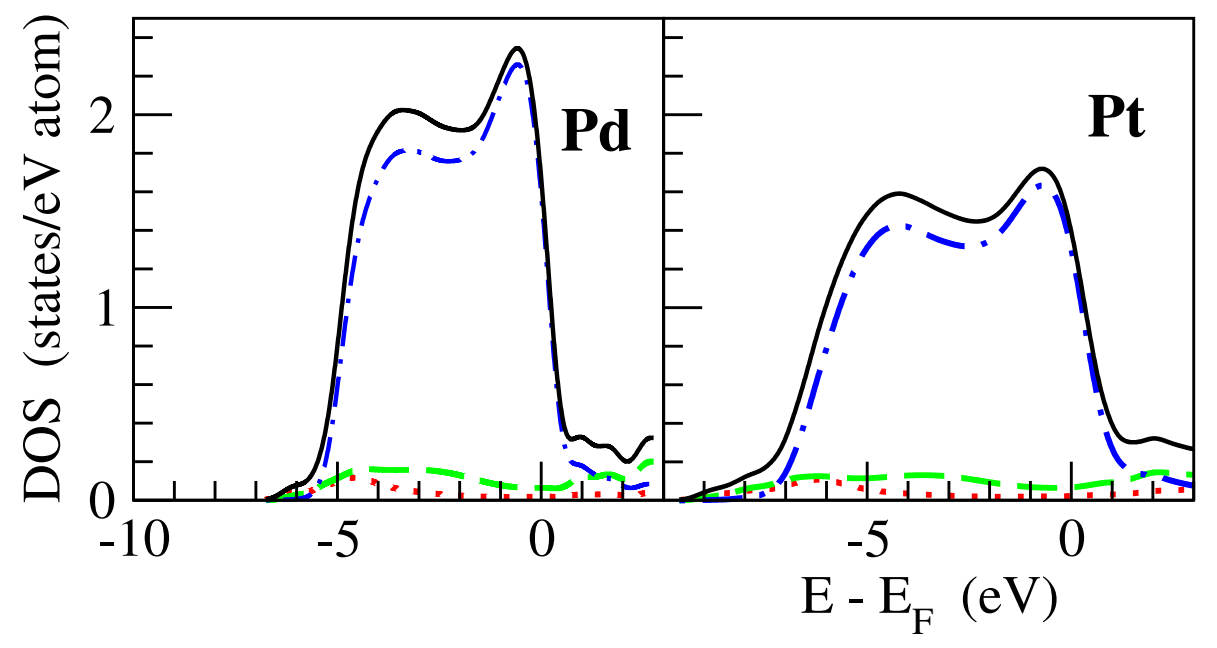

Figure 11. Total electronic density of states (black full line) for l-Pd and l-Pt near their respective melting points, as obtained from the present AIMD calculations. The angular decomposition of the DOS in $s$ (red dotted line), $p$ (green dashed line) and $d$ (blue dash-dotted line) is also shown.

five ionic configurations separated from each other by around $20 \mathrm{ps}$, and sampling over the Brillouin zone of the cubic simulation cell using a $6 \times 6 \times 6$ Monkhorst-Pack grid.

The results obtained for the single-particle partial and total $n(E)$ associated to the outer ten valence electrons are plotted in figure 11. Obviously, the main contribution comes from the $d$ states, with a very small contribution from the $s$ states. For both systems, the corresponding $n(E)$ clearly point to a metallic character.

The most obvious aspect in the comparison between the DOS in Pd and $\mathrm{Pt}$ is the increased width in the $d$-band, and therefore in the whole DOS, of l-Pt. The main reason for this increased width is the larger overlap among the $d$-states of different atoms, due to the larger extent of the $d$ atomic orbital in $\mathrm{Pt}$ as compared with $\mathrm{Pd}$, combined with an almost identical typical separation between near neighbours in both liquid metals. Note that the DOS of the fcc solids, both in Pd and Pt [13], have similar characteristics as those found here for the melts, except that our results are more smeared due to disorder.

The relative differences between the DOS's in Pd and Pt could show up directly as differences in their electrical conductivities and optical properties, which are not discussed in this paper, since they would require a more thorough treatment of electronic excited states and, at least in the case of $\mathrm{Pt}$, an analysis of spin-orbit effects. The magnitudes considered here, static and dynamic, are a consequence of the forces acting on the atoms, which themselves are determined from the electronic properties, since the potential energy for atomic motion is obtained in terms of sums over states up to the Fermi level. However, these are integrated magnitudes and the detailed differences in DOS need not show up explicitly and directly in the atomic accelerations that drive the dynamics of the systems.

So, the electronic properties for given atomic positions determine the forces, these forces drive the dynamics changing the atomic positions, and the new atomic positions generate new electronic properties, and so on. The atomic dynamics and the 


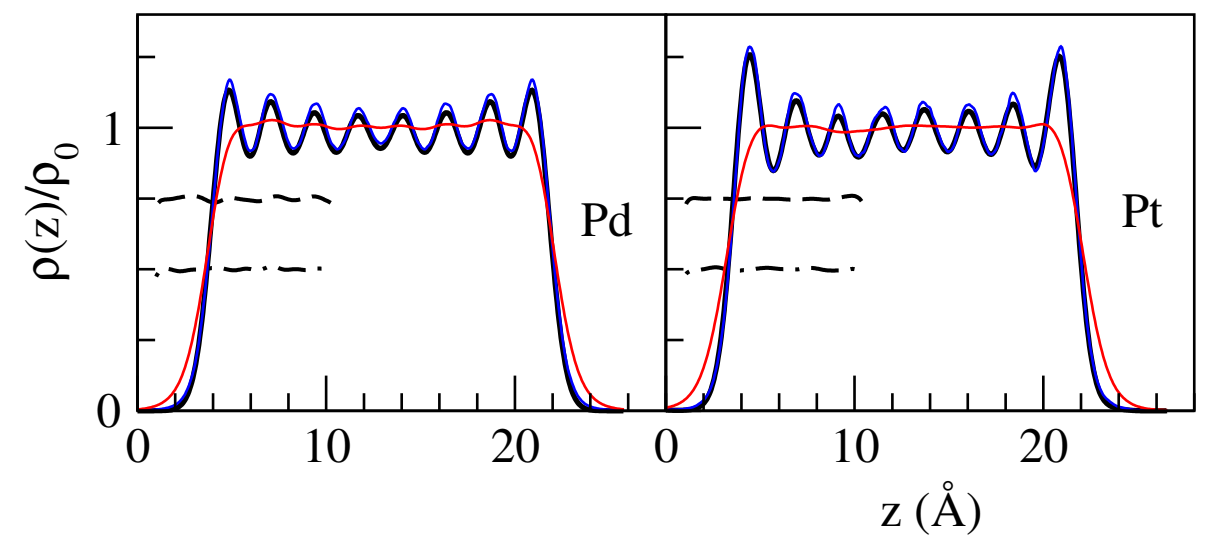

Figure 12. Ionic (thick black line), valence electronic (red line) and total electronic (core+valence) (blue line) density profiles of the slabs of l-Pd (top) and l-Pt (bottom) at 2000 and $2200 \mathrm{~K}$ respectively. All the densities have been plotted relative to the respective bulk values. The dashed and dot-dashed lines are the $x$-transverse (displaced by -0.25) and the $y$-transverse (displaced by -0.50 ) ionic density profiles.

electronic properties are so closely interwoven that none can be considered simply as a consequence of the other.

\section{Results: Free Liquid Surface}

We report some structural results for the FLS of l-Pd and l-Pt at $T=2000$ and 2200 $\mathrm{K}$ respectively. For both systems, the AIMD simulations were performed with 120 atoms in a slab geometry with dimensions given in section 2. After thermalization, we generated around 30000 ionic configurations (see section 2) which were used to analyze the slab's properties.

Figure 12 shows the longitudinal ionic density profiles (DP) obtained, where we observe a clear-cut stratification, with a larger amplitude of the oscillations at the outer layers. The height of the maxima of the DP in the outermost layers is larger for l-Pt than for l-Pd. As for the wavelength of the oscillations, we find that for l-Pd the spacing between two consecutive maxima remains constant to a value $\approx 2.30 \pm 0.03$ $\AA$. This same value applies for the wavelength of the inner oscillations in l-Pt, but a somewhat greater value of $\approx 2.45 \pm 0.03 \AA$ is obtained for the spacing between the outer and the first inner layer. For comparison, we have also evaluated the transverse ionic DP's which have also been included in Fig. 12. Notice the very weak oscillatory shape along with substantially smaller amplitudes.

It is worth noting that, according to experiments [44, 45, 46, 47] and AIMD simulations [19, 20, 22, 23, 48], most liquid metals have an oscillating ionic DP with a single wavelength. So, the apearance of two different wavelengths in the ionic DP is remarkable because this feature has only been found, so far, in $\mathrm{l}-\mathrm{Hg}, \mathrm{l}-\mathrm{Cd}$ and $\mathrm{l}-$ $\mathrm{Ag}$ near their respective triple point $[16,17,23,44]$. Furthermore, whereas $\mathrm{l}-\mathrm{Hg}$ and l-Ag show a similar behavior as l-Pt, l-Cd shows an opposite trend because its outer wavelength was found to be smaller than the inner ones.

A more detailed analysis of the different physical properties inside the slab is 


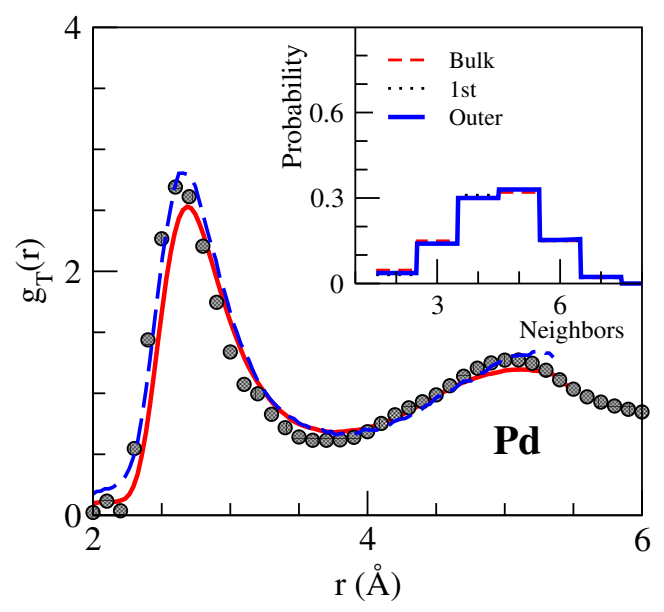

Figure 13. Transverse pair correlation function in a central slice (full red line) and at the outer slice (dashed blue line) for l-Pd at $2000 \mathrm{~K}$. The grey circles stand for the bulk XD data of Waseda [1] at $1853 \mathrm{~K}$. The inset shows the distribution of the number of in-plane neighbors for different regions of the slab.

facilitated by partitioning the slab into slices ( 8 of them) located between consecutive minima of the longitudinal ionic DP, with the outer slices comprising between the outermost minima and the points at the decaying tails where the density takes half its bulk value.

First, we found that the average ionic number density remained practically the same for all the slices of the slab of l-Pd, whereas in the case of l-Pt the outer slices showed a density increase of $\approx 5.0 \%$ with respect to the central slices. A change in the ionic number density of the outer slices has also been observed in several liquid metals [23, 48], with a density decrease in some cases (alkalis and alkali-earths) and a density increase in others $(\mathrm{Hg}, \mathrm{Cd}, \mathrm{Al}, \mathrm{Ga}, \mathrm{In}, \mathrm{Tl}, \mathrm{Si}, \mathrm{Sn})$.

The transverse pair distribution function, $g_{T}(r)$, describes the probability of finding two particles within the same layer separated by a distance $r$, being therefore a quasi-twodimensional function. Figures 13-14 show the calculated $g_{T}(r)$ for the central and outer slices in the slabs of l-Pd and l-Pt. As expected, the $g_{T}(r)$ in the central slices is very similar to the experimental bulk $g(r)$, but in the outer slice the corresponding $g_{T}(r)$ shows some rearrangement of the surface atoms. Whereas for l-Pd the surface $g_{T}(r)$ hardly changes with respect to the inner slices, for l-Pt the surface $g_{T}(r)$ is slightly displaced to smaller $r$-values and its main peak is higher. Notice that this correlates with the small increase in the average density of the outer slices for l-Pt and the invariance of the average density of the outer slices of l-Pd.

We consider two atoms within the same layer as in-plane neighbors when they are separated by a distance smaller than the minimum of the corresponding $g_{T}(r)$. Simply by counting the number of in-plane neighbors of all the atoms of the selected layer during the simulation run we can obtain their distribution. The insets of figures 1314 depict the results obtained for l-Pd and l-Pt respectively. The results correspond to a central slice ("bulk" values), the outer slice and the first inner slice. In the bulk region, both for l-Pd and l-Pt, four-fold and five-fold in-plane coordination are equally probable. The same situation holds at the first inner slices for both systems. 


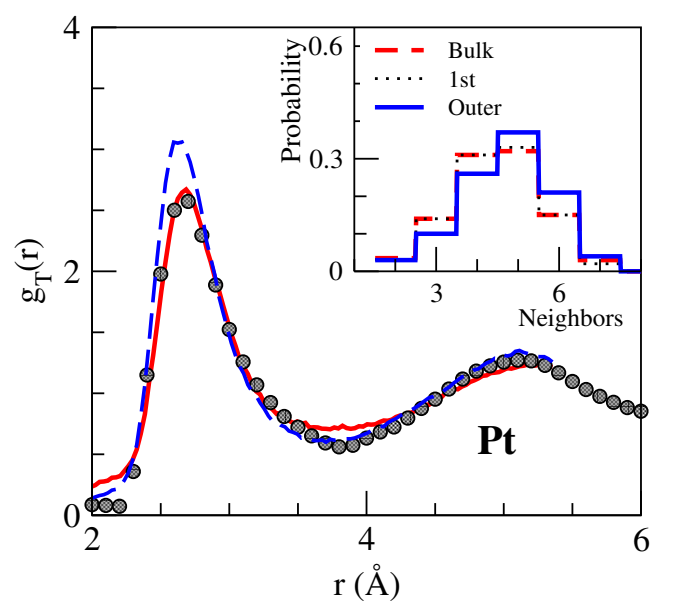

Figure 14. Same as the previous figure, but for l-Pt at $2200 \mathrm{~K}$. The grey circles stand for the bulk x-ray diffraction data of Waseda [1] at $2053 \mathrm{~K}$.

Moreover, the distribution does not change either at the outermost layers in l-Pd. It is only at the outermost layers of l-Pt where a certain redistribution is observed, with an increase in the probability of five-fold coordination (around $35 \%$ of the atoms) and a decrease in the corresponding probability of four-fold coordinated atoms (around 28 $\%$ ). Again this correlates with the changes in the values of the average ionic density at the outer slice.

The structure of the FLS can in principle be determined by x-ray reflectivity experiments, which provide information about the total electronic density, including core and valence electrons, within a range of several angstroms inside the liquid. We briefly describe the formulation below and further details can be obtained, for instance, in Ref. [51]. The measured experimental reflectivity, $R_{\exp }\left(q_{z}\right)$, is decomposable into several terms,

$$
\begin{aligned}
R_{\exp }\left(q_{z}\right) & =R_{F}\left(q_{z}\right)\left|\Phi_{\exp }\left(q_{z}\right)\right|^{2} \\
& =R_{F}\left(q_{z}\right)\left|\Phi_{\text {int }}\left(q_{z}\right)\right|^{2} \exp \left(-\sigma_{c, \exp }^{2} q_{z}^{2}\right)
\end{aligned}
$$

where $q_{z}$ is the momentum transfer perpendicular to the FLS. The factors appearing in equation (3) are the following: $R_{F}\left(q_{z}\right)$ is the Fresnel reflectivity of a perfectly sharp step-function interface; $\Phi_{\exp }\left(q_{z}\right)$ is the surface structure factor, which is related to the average density profile; $\Phi_{\text {int }}\left(q_{z}\right)$ is the intrinsic surface structure factor, defined below, that is related to the layered structure that would show the interface if there were no thermally excited capillary waves (the so called intrinsic density profile); and finally the last term expresses the effect produced by the capillary waves that, inevitably, are present.

The most interesting term, whose determination is the aim of the measurements, is the intrinsic surface structure factor. This is defined as the FT of the derivative of the intrinsic normalized total electron density profile (TEDP), $n_{e, \text { int }}^{T}(z) / n_{e 0}^{T}$,

$$
\Phi_{\text {int }}\left(q_{z}\right)=\frac{1}{n_{e 0}^{T}} \int_{-\infty}^{\infty}\left(\frac{\partial n_{e, \text { int }}^{T}(z)}{\partial z}\right) \exp \left(i q_{z} z\right) d z .
$$




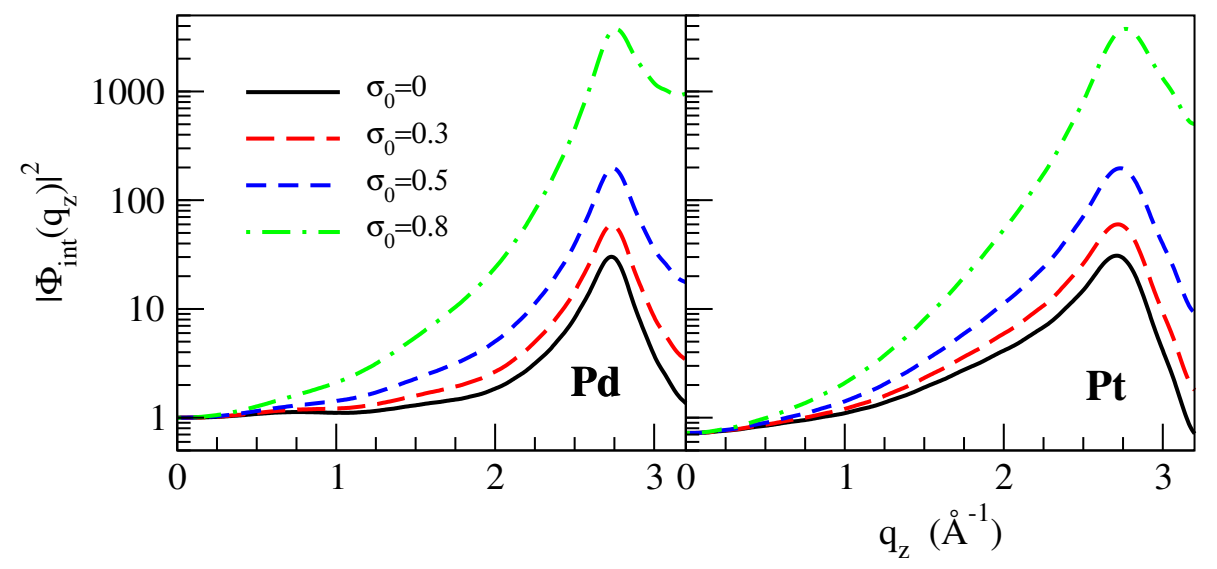

Figure 15. Squared modulus of the intrinsic surface structure factor of l-Pd and l-Pt free liquid surfaces, obtained from their respective calculated total electronic density profile. The curves correspond to different choices of $\sigma_{0}$ (in $\AA$ ).

The determination of $\left|\Phi_{\text {int }}\left(q_{z}\right)\right|^{2}$ from the measured reflectivity requires the use of an explicit value for $\sigma_{c, \exp }$. This is usually decomposed into two contributions, $\sigma_{c, \exp }^{2}=\sigma_{0}^{2}+\sigma_{\mathrm{cw}, \exp }^{2}$, where $\sigma_{0}$ represents an intrinsic surface roughness, whose origin is still under debate, but which has proved to be essential for an accurate description of the experimental reflectivity data $[44,45,46,47,49,50,51]$. As for $\sigma_{\mathrm{cw}, \exp }$, it is obained within the macroscopic capillary wave theory, in terms of the surface tension, the temperature, the atomic size and the size of the sample's surface illuminated by the $\mathrm{x}$-rays in the experimetal setup.

A similar approach can be followed to predict the intrinsic surface structure factor corresponding to the AIMD simulations [16, 23, 49]. The most important difference now is that the size is determined by the simulation box, which is much smaller than the illuminated size in the experiments. This leads to smaller values for the capillary roughness $\sigma_{\mathrm{cw}, \mathrm{AIMD}}$ as compared to experiments, $\sigma_{\mathrm{cw} \text {,exp }}$. The values obtained for $\sigma_{\mathrm{cw}, \mathrm{AIMD}}$ are 0.58 and $0.63 \AA$ for $\mathrm{Pd}$ and $\mathrm{Pt}$ respectively. Since there is no clearcut definition of the intrinsic roughness, $\sigma_{0}$, we have computed the intrinsic surface structure factors that correspond to values of $\sigma_{0}=0,0.3,0.5$ and $0.8 \AA$.

The only input needed to compute the AIMD intrinsic structure factor is then the total electronic density profile. This is the sum of the density profile due to valence electrons, which is computed selfconsistently during the simulation run, and the density profile due to core electrons, which we obtain by superposing atomic core densities at the atomic positions obtained along the simulation.

The valence electron density profiles for Pd and Pt are shown (normalized) in figure 12, where we can observe that the corresponding oscillations are very small in amplitude. The total electronic density profiles are also shown (normalized) in figure 12, and are quite similar to the ionic density profile, basically because they are dominated by the core component (36 vs 10 electrons in $\mathrm{Pd}, 68$ vs 10 electrons in $\mathrm{Pt}$ ).

The calculated intrinsic surface structure factors, are depicted in Fig. 15 for different choices of $\sigma_{0}$. For both metals, we observe that all the curves have a main Bragg-like peak at $q_{z} \approx 2.74 \AA^{-1}(\mathrm{l}-\mathrm{Pd})$ and $\approx 2.71 \AA^{-1}(\mathrm{l}-\mathrm{Pt})$. These values are close to $2 \pi / \lambda$ and are related to the stratification surface layering exhibited by their 
respective longitudinal TEDP which in both cases had a wavelength $\lambda \approx 2.30 \AA$. However, the fact that l-Pt had a slightly larger wavelength at the outermost slice may explain the slightly smaller value for its peak's position. For smaller $q_{z}$, the $\left|\Phi_{\text {int }}\left(q_{z}\right)\right|^{2}$ decreases uniformly towards its value at $q_{z}=0$. The shape and behavior of both curves is qualitatively similar to the experimental data obtained for other liquid metals such as $\mathrm{Hg}$, Ga, In, and $\mathrm{K}$ [44, 45, 46, 47].

\section{Conclusions}

An ab-initio molecular dynamics simulation method has been used to calculate several bulk properties as well as the free liquid surface of l-Pd and l-Pt at thermodynamic states near their respective triple point. These two metals have not attracted much attention yet and therefore there is not much theoretical and/or experimental information to compare with.

As for the bulk static structure, the $a b$ initio results for the pair distribution function, $g(r)$, and the static structure factor, $S(q)$, are in good agreement with their respective experimental XD data. A more detailed study of the liquid local structure, by using the CNA method, showed in both systems a substantial presence of perfect and distorted icosahedral structures.

The intermediate scattering functions, $F(q, t)$, show at small $q$-values, strong oscillations superposed on a distinct diffusive component. Moreover, starting from the calculated $F(q, t)$ we have analyzed the decay modes associated with the relaxations of the collective excitations. The procedure was to evaluate the associated secondorder memory function which was subsequently fitted to a model comprising two exponentially decaying functions intended to describe a fast and a slow relaxation processes. The analysis concerning the physical origin of both relaxation processes showed that a proper description of the microscopic dynamics of both l-Pd and l-Pt is provided by a generalized hydrodynamic model.

The AIMD dynamic structure factors, $S(q, \omega)$, show side-peaks which are indicative of collective density excitations. Futhermore, a detailed analysis of the $S(q, \omega)$ has revealed the same type of transverse-like excitation modes as experimentally found in some other liquid metals.

For both systems studied, the corresponding transverse dispersion relation exhibits two branches with the high-frequency branch existing over a limited $q$-range. This new, high-frequency branch, has a frequency which practically coincides with that of the shoulder appearing in the corresponding velocity autocorrelation function.

Several transport coefficients such as self-diffusion, adiabatic sound velocities and shear viscosities have also been evaluated and they show good agreement with the available experimental data.

We have also presented results for the free liquid surface of 1-Pd and l-Pd, for which no experimental data are available yet. For both metals, we obtain surface layering which is a feature exhibited by several other liquid metals. Furthermore, we have performed a calculation of the intrinsic surface stucture factor which is the magnitude measured in an x-ray reflectivity experiment. For both l-Pd and l-Pt, the obtained results show a behavior which is qualitatively very similar to what has already been found in several other liquid metals. 
Liquid palladium and platinum: bulk and surface properties.

\section{Acknowledgments}

We acknlowledge the funding from Junta de Castilla y Leon (project VA124G18). LEG and DJG additionally acknowledge the support of the Spanish Ministry of Economy and Competitiveness (Project PGC2018-093745-B-I00), and its partial support by european FEDER funds.

\section{References}

[1] Waseda Y 1980 The Structure of Non-Crystalline Materials, (New York: McGraw-Hill)

[2] Blairs S 2006 J. Colloid Interface Sci. 302312 Blairs S 2007 Int. Materials Rev. 52321 Blairs S 2007 Phys. Chem. Liq. 45399

[3] Iida T and Guthrie R I L 2015 The Thermophysical Properties of Metallic Liquids (Oxford: Oxford Univ. Press)

[4] Alemany M M G, Rey C and Gallego L J 1998 J. Chem. Phys. 1095175 Alemany M M G, Rey C and Gallego L J 1999 Phys. Rev. B 609208

[5] Kart S O, Tomak M, Uludogan M and Cagin T 2004 J. Non-Cryst. Solids 337101

[6] Hohenberg P and Kohn W 1964 Phys. Rev. 136 B864 Kohn W and Sham L J 1965 Phys. Rev. 140 A1133

[7] del Rio B G, González L E and González D J 2017 J. Chem. Phys. 146034501

[8] Gianozzi P et al 2009 J. Phys.: Condens. Matter 21395502 Gianozzi P et al 2017 J. Phys.: Condens. Matter 29465901

[9] Ceperley D M and Alder B J 1980 Phys. Rev. Lett. 45566 Perdew J P and Zunger A 1981 Phys. Rev. B 235048

[10] Louie S G, Froyen S and Cohen M L 1982 Phys. Rev. B 261738

[11] Vanderbilt D 1990 Phys. Rev. B 417892.

[12] Haas P, Tran F and Blaha P 2009 Phys. Rev. B 79085104.

[13] Ahmed S, Zafar M, Shakil M and Choudhary M A 2016 Chin. Phys. B 25036501.

[14] Calderin L, González D J, González L E and López J M 2008 J. Chem. Phys. 129194506

[15] Calderín L, González L E and González D J 2009 J. Chem. Phys. 130194505 Calderín L, González L E and González D J 2011 J. Phys.: Condens. Matter 23375105

[16] Calderín L, González L E and González D J 2013 J. Phys.: Condens. Matter 25065102

[17] del Rio B G, González L E and González D J 2016 Phys. Fluids 28107105

[18] del Rio B G, Rodriguez O, González L E and González D J 2017 Comp. Mater. Science 139 243

[19] Walker B G, Molteni C and Marzari N 2004 J. Phys.: Condens. Matter 16 S2575 Walker B G, Marzari N and Molteni C 2006 J. Chem. Phys. 124174702 Walker B G, Marzari N and Molteni C 2007 J. Chem. Phys. 127134703

[20] Fabricius G, Artacho E, Sanchez-Portal D, Ordejon P Drabold D A and Soler J M 1999 Phys. Rev. B 60 R16283

[21] Salomons E and Mareschal M 1991 J. Phys: Condens. Matter 33645 Sides S W, Grest G S and Lacasse M D 1999 Phys. Rev. E 606708

[22] González D J, González L E and Stott M J 2004 Phys. Rev. Lett. 92, 085501 (2004) González D J, González L E and Stott M J 2005 Phys. Rev. Lett. 94077801

[23] Calderín L, González L E and González D J 2009 Phys. Rev. B 80115403 Calderín L, González L E and González D J 2013 Phys. Rev. B 87014201

[24] Balucani U and Zoppi M 1994 Dynamics of the Liquid State (Oxford: Clarendon)

[25] Honeycutt J D and Andersen H C 1987 J. Phys. Chem. 914950

[26] Marcus Y 2017 J. Chem. Thermodynamics 10911

[27] Bafile U, Guarini E and Barocchi F 2006 Phys. Rev. E 73061203

[28] Scopigno T, Balucani U, Ruocco G and Sette F 2000 J. Phys.: Condens. Matter 128009

[29] Canales M, González L E and Padró J A 1994 Phys. Rev. E 503656 Bryk T and Mryglod I 2000 J. Phys.: Condens. Matter 123543 Bryk T and Mryglod I 2001 J. Phys.: Condens. Matter 131343 Bryk T and Mryglod I 2001 Phys. Rev. E 63051202

[30] Hosokawa S, Inui M, Matsuda K, Ishikawa D and Baron A Q R 2008 Phys. Rev. B 77174203

[31] Bryk T, Ruocco G, Scopigno T and Seitsonen A P 2015 J. Chem. Phys. 143110204

[32] Marqués M, González D J and González L E 2016 Phys. Rev. B 94024204 
Liquid palladium and platinum: bulk and surface properties.

Marqués M, González L E and González D J 2016 J. Phys.: Condens. Matter 28075101

[33] Bryk T, Demchuk T and Jakse N 2019 Phys. Rev. B 99014201

[34] Jakse N and Bryk T 2019 J. Chem. Phys. 151034506

[35] Bryk T, Demchuk T, Jakse N and Wax J F 2018 Frontiers in Physics 600006

[36] del Rio B G and González L E 2017 Phys. Rev. B 95224201

[37] del Rio B G, Chen M, González L E and Carter E A 2018 J. Chem. Phys. 149094504

[38] Palmer B J 1994 Phys. Rev. E 49359

[39] Balucani U, Brodholt J P, Jedlovszky P and Vallauri R 2000 Phys. Rev. E 622971 Blanco J, González D J, González L E, López J M and Stott M J 2003 Phys. Rev. E 67 041204

[40] Hosokawa S et al 2009 Phys. Rev. Lett. 102105502 Hosokawa S et al 2011 Eur. Phys. J. Special Topics 19685

[41] Hosokawa S, Munejiri S, Inui M, Kajihara Y, Pilgrim W-C, Baron A Q R, Shimojo F and Hoshino K 2013 AIP Conf. Proc. 1518695

Hosokawa S, Munejiri S, Inui M, Kajihara Y, Pilgrim W-C, Ohmasa Y, Tsutsui S, Baron A Q R, Shimojo F and Hoshino K 2013 J. Phys.: Condens. Matter 25112101

Hosokawa S, Inui M, Kajihara Y, Tsutsui S and Baron A Q R 2015 J. Phys.: Condens. Matter 27194104

[42] Giordano V M and Monaco G 2010 Proc. Natl. Acad. Sci. USA 10721985

[43] Zanatta M, Sacchetti F, Guarini E, Orecchini A, Paciaroni A Sani L and Petrillo C 2015 Phys. Rev. Lett. 114187801

[44] Magnussen O M, Ocko B M, Regan M J, Penanen K, Pershan P S and Deutsch M 1995 Phys. Rev. Lett. $\mathbf{7 4} 4444$

[45] Regan M J, Kawamoto E H, Lee S, Pershan P S, Maskil N, Deutsch M, Magnussen O M, Ocko B M and Berman L E 1995 Phys. Rev. Lett. 752498

[46] Tostmann H, DiMasi E, Pershan P S, Ocko B M, Shpyrko O G and Deutsch M 1999 Phys. Rev. B 59783

[47] Shpyrko O G, Huber P, Grigoriev A Y, Pershan P S, Ocko B, Tostmann H and Deutsch M 2003 Phys. Rev. B 67115405

[48] González D J, González L E and Stott M J 2006 Phys. Rev. B, 74014207

[49] González D J and González L E 2008 J. Phys.: Condens. Matter 20114118

[50] Pershan P S, Stoltz S E, Shpyrko O G, Deutsch M, Balagurusamy V S K, Meron M, Lin B and Streitel R 2009 Phys. Rev. B 79115417

[51] Pershan P S and Schlossman M L 2012 Liquid Surfaces and Interfaces. Synchrotron X-ray Methods, (New York: Cambridge) 